\title{
The Active Embedded Ubiquitous Web Service Framework
}

\author{
Dugki Min ${ }^{1, *}$, Junggyum Lee ${ }^{1}$, and Eunmi Choi ${ }^{2, * *}$ \\ ${ }^{1}$ School of Computer Science and Engineering, Konkuk University \\ Hwayang-dong, Kwangjin-gu, Seoul, 143-701, Korea \\ dkmin@konkuk.ac.kr, stutle@dms.konkuk.ac.kr \\ ${ }^{2}$ School of Business IT, Kookmin University \\ Chongnung-dong, Songbuk-gu, Seoul, 136-702, Korea \\ emchoi@kookmin.ac.kr
}

\begin{abstract}
We develop an active embedded middleware framework, called the EUWS (Embedded Ubiquitous Web Service), in WinCE.NET. The EUWS seamlessly integrates home network services and the Web Services on the Internet and provides a management framework for ubiquitous web services. As the initial stage of our project, our current focus has been on designing and implementing a prototype of the EUWS in WinCE.NET. The architecture of the EUWS prototype system includes an extensible and reconfigurable Embedded Ubiquitous Web Service(EUWS) framework and an UPnP2WS processing module that seamlessly integrates the UPnP standard with the Web Service standard.
\end{abstract}

\section{Introduction}

Recently, a number of middleware standards are proposed to implement home network. They are the UPnP(Universal Plug and Play)[1] for easy interoperability among devices, the HAVi(Home Audio and Video Interoperability)[2] for interoperability between video and audio devices, the Jini[3] for interoperability for Java applications, and the OSGI[4] for middleware framework between networked services. These recent home network middlewares are used to connect, integrate, and manage services provided by devices that are in a restricted area. However, none of them considers the seamless interconnection and integration with the external Internet services, i.e., to access home network services from the external client or to reach out the external Internet services from home network services.

As the standard technology to integrate Internet services, the Web Service[5] becomes the major trend. The Web Service is platform-independent and programming languageindependent, and it is the XML-based middleware standard determined and developed by

* This paper was supported by Konkuk University in 2005", and also by Microsoft Research Asia under the Grant of MSRA Joint Research Project in 2004.

**Corresponding author: This work was supported the Korea Research Foundation (KRF) under Grant No. D00021 (R04-2003-000-10213-0), and also by research program and research center UICRC of Kookmin University in 2005. 
most of IT vendors. The Web Service provides the fundamental middleware standards of Internet distributed computing: SOAP[6] for the communication standard between services, WSDL[7] for description standard to define the Web Service in XML, UDDI[8] for service searching standard over Internet. Moreover, it extends up to the SOA-based application middleware standard by considering security, transaction, event, and business process management.

We develop an active embedded middleware framework, called the EUWS (Embedded Ubiquitous Web Service), in WinCE.NET that can be applied to home gateways. The EUWS seamlessly integrates home network services, and the Web Services on the Internet and provides a management framework for ubiquitous web services. Through the EUWS home gateway, the device services are converted to the Web Services for external accesses, so that a remote client can control the device via the Web Services. At the same time, the EUWS gateway converts the external Web Services into the device-specific services, so that the internal devices can access and use the external services according to the device-specific protocol.

As the initial stage of our research, our current focus has been on designing and implementing a prototype of the EUWS in WinCE.NET for the UPnP middleware protocol. The architecture of the EUWS prototype system includes an extensible and reconfigurable Embedded Ubiquitous Web Service(EUWS) framework and a UPnP2WS processing module that seamlessly integrates the UPnP standard with the Web Service standard. The EUWS technology will be used as an encore technology to build the Advanced Home Gateway. We have the EUWS prototype be deployed into a home gateway in our demo system where a digital audio and a digital TV can be controlled by an external remote controller. While the traditional gateway focuses on connection between internal segment and external segment at the network level, the EUWS focuses on connection between internal services and external services at the service level. The EUWS integrates various services which exist in home network environment, and integrates internal and external services.

The next section presents the EUWS architecture. Section 3 presents our demo prototype system currently implemented. We conclude in the last section.

\section{The EUWS Architecture}

In this section, we introduce the architecture of the active EUWS (Embedded Ubiquitous Web Service) framework. As shown in Figure 1, the active EUWS framework has two major parts: one is the Protocol Abstraction Sub-framework and the other is the Service Orientation Sub-framework. The Protocol Abstraction Subframework is for seamlessly integrating various devices each of which uses different middleware protocols. The Service Orientation Sub-framework is for creating a virtual service-oriented space where everything in a ubiquitous environment, e.g. devices or services, is a standard service.

\subsection{Protocol Abstraction Sub-framework}

In order to support various ubiquitous devices that use different communication protocols, the Protocol Abstraction Sub-framework contains an Active Protocol 


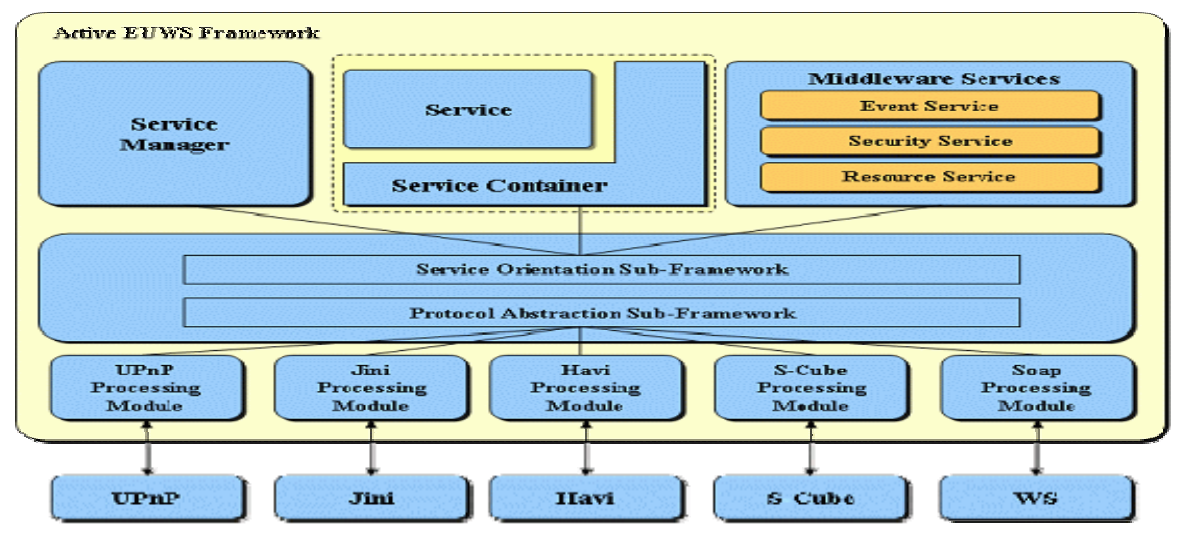

Fig. 1. The EUWS Framework Architecture

Reconfigurable architecture that can dynamically deploy protocol processing modules on demand. The active protocol reconfigurable architecture is composed of two dedicated modules and one or more pluggable modules. The two dedicated modules are the Protocol Detection Module and the Dynamic Protocol Deployment Module.

The Protocol Detection Module is used for detecting new devices whose protocol processing module is not yet plugged-in. While the EUWS framework is running, the Protocol Detection Module periodically broadcasts a sequence of protocol-specific discovery messages one-by-one at a time. When it receives any response from a new device whose protocol execution module is not yet plugged-in, the related protocol processing module is downloaded and deployed automatically by the Protocol Deployment Module, if the related protocol processing module is available. Otherwise, error message is sent to the newly detected devices. The Protocol Detection Module can be upgraded whenever its new version is available.

\subsection{Service Orientation Sub-framework}

The Service Orientation Sub-framework provides a service orientation environment where every distinctive device or service is recognized and treated as the same type of standard service. This framework also provides common middleware services, such as resource management service, event service, and security service. In our project, we employ the Web service as our service-orientation standard, since the Web service becomes the de-facto standard of business domain service area, and is also good at extensibility and self-description.

The Service Orientation Sub-framework has three components: Service Manager, Service Container, and Middleware Services. The Service Manager is the manager of service orientation; it is in charge of transforming everything to standard service, registering and searching a registered service to the service directory, and providing metadata information of the registered services. Let us suppose that an UPnP device is newly arrived into the managed space. Then, the UPnP Processing Module detects the UPnP device and registers it to the Service Manager. The Service Manager creates a service proxy that acts as the corresponding service object for the device and uploads 
it to the Service Container. At the same time, the WSDL of the service proxy is automatically generated. After the service proxy is deployed with WSDL into the Service Container, the Service Manager registers the device as a Web service into the UDDI. Other web services, which locate outside of the framework, consider the UPnP device as a registered Web service. Within our framework, this device is also treated as a standard service. That is, the Protocol Execution Module or the Service Manager accesses a registered device through the corresponding service proxy. However, external devices, that use their own protocols, access and use the registered device through their own protocols via the related Protocol Execution Modules.

Other functions of the Service Orientation Sub-framework are the Middleware Services related to Service Container. The Service Container is in charge of system resource management and dynamic service deployment into memory if necessary. Also, the Service Container performs various management functions by detecting service invocation and generating events.

\section{EUWS Prototype Implementation}

In this section, we introduce the EUWS prototype implemented. The EUWS prototype is a system operated on .NET framework and it contains the initial version of EUWS Framework with the UPnP execution module. In order to deploy the EUWS into a device, Win CE .NET Platform Builder is used to upload a kernel image into the board.

\subsection{Development Environment}

The EUWS is implemented in an embedded board on top of Win CE .NET containing .NET Compact Framework. Two embedded boards are used; one is for a device and the other is for home gateway. The prototype device and home gateway are implemented on the similar boards called X-Hyper255B and X-Hyper255BTKUIII respectively. These boards have a $400 \mathrm{MHz}$ Intel XScale PXA255 CPU, 64MB SDRAM, 32MB flash memory, 10Base-T CS8900A and PCMCIA Slot. Detailed information is in Table 1. As for the external control client, HP iPAQ PDA is used. As a design tool, the IBM Rational Rose XDE is used, and as a development tool, the MS Visual Studio .NET is used.

Table 1. Device Platform \& Home Gateway Platform

\begin{tabular}{|l|l|}
\hline Device Platform & Home Gateway Platform \\
\hline and & \\
\hline
\end{tabular}

\subsection{Devices of Demo System}

As our demo systems, a digital audio and a digital TV are developed as UPnP devices (see Figure 2) Those devices contain UPnP device modules deployed on top of .NET 
Compact Framework. When initially operating, their device information is transferred to the home gateway. Also, they have UPnP control point modules so that they can access web services provided by the home gateway as UPnP services. For example, the digital TV can receive channel broadcasting information service from the home gateway, and the digital audio can receive new media information.
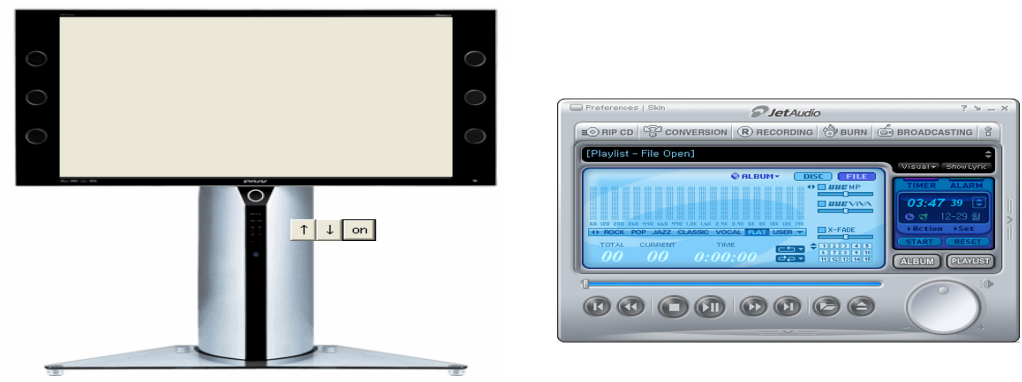

Fig. 2. UPnP Devices (Digital TV \& Digital Audio)

The home gateway contains the active EUWS framework explained in section 2. It discovers internal devices, and converts the services of the internal devices into Web services. It also converts external Web services into UPnP services. At the same time, the home gateway reads events from UPnP devices and transfers them to external clients. Especially, when an external Web service is accessed by an UPnP device, the external Web service is perceived as an UPnP service by the EUWS framework of the home gateway, that is an UPnP device including an UPnP control point can access the external Web Services as easy as UPnP services via the home gateway.

In order to control digital audio and TV devices within home area, a PDA is used as external device that contains the home control application. When booting the PDA, the control application automatically begins and receives service information of home devices from the designated home gateway. The device information received by the home control application is written in the Web services of internal UPnP devices exported by the home gateway. Thus, when a user selects and invokes a service shown in the screen of the PDA, it invokes a Web service provided by the home gateway and it consequently invokes an UPnP service provided by an internal device. The Figure 3 shows the GUI of the PDA.
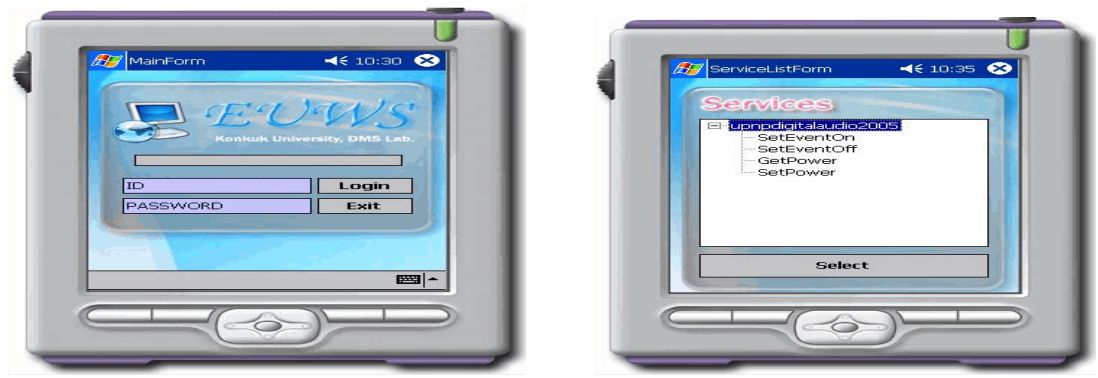

Fig. 3. External Devices 


\section{Conclusion}

In order to establish a ubiquitous home network, we developed an active embedded middleware framework, the EUWS, in WinCE.NET environment. The EUWS seamlessly integrates home network services and the Web Services on the Internet, and also provides a management framework for ubiquitous web services. In this paper, we presented a prototype of the EUWS in WinCE.NET for the UPnP middleware protocol, so that we can seamlessly integrates the UPnP standard with the Web Service standard, and work with UPnP devices and home gateway for the home network. Through our demo system, we could control a digital audio and a digital TV by an external remote controller. As an active embedded middleware framework, the EUWS achieved to integrate various existing services, internal and external services.

\section{References}

[1] Miller, B.A., Nixon, T., Tai, C., Wood, M.D.: Home networking with Universal Plug and Play. Communications Magazine 39(12), 104-109 (2001)

[2] HAVI, http: / /www.havi.org

[3] Allard, J., Chinta, V., Gundala, S., Richard III, G.G.: Jini meets UPnP: an architecture for Jini/UPnP interoperability. In: Proceedings. 2003 Symposium on Applications and the Internet, pp. 268-275, January 27-31 (2003)

[4] Dobrev, P., Famolari, D., Kurzke, C., Miller, B.A.: Device and service discovery in home networks with OSGi. Communications Magazine 40(8), 86-92 (2002)

[5] Hung, P.C.K., Ferrari, E., Carminati, B.: Towards standardized Web services privacy technologies. In: Proceedings IEEE International Conference on Web Services, p. 174, July 6-9 (2004)

[6] Box, D.: SOAP 1.1., http: / / www.w3 .org/TR/SOAP/

[7] WSDL Verstion 2.0 Part 3: Bindings, http://www.w3.org/TR/wsdl20bindings /

[8] UDDI Technical WhitePaper, UDDI.org, http: / /www. uddi.org/whitepapers.html 